\title{
EQUIVALENT GROUPOIDS HAVE MORITA EQUIVALENT STEINBERG ALGEBRAS
}

\author{
LISA ORLOFF CLARK AND AIDAN SIMS
}

\begin{abstract}
Let $G$ and $H$ be Hausdorff ample groupoids and let $R$ be a commutative unital ring. We show that if $G$ and $H$ are equivalent in the sense of Muhly-RenaultWilliams, then the associated Steinberg algebras of locally constant $R$-valued functions with compact support are Morita equivalent. We deduce that collapsing a "collapsible subgraph" of a directed graph in the sense of Crisp and Gow does not change the Morita-equivalence class of the associated Leavitt path $R$-algebra, and therefore a number of graphical constructions which yield Morita equivalent $C^{*}$-algebras also yield Morita equivalent Leavitt path algebras.
\end{abstract}

\section{INTRODUCTION}

Two groupoids $G$ and $H$ are equivalent if they act freely and properly on the left and right (respectively) of a space $Z$ in such a way that the quotient of $Z$ by the action of $G$ is homeomorphic to the unit space of $H$ and vice versa. It was shown in [14] that if second-countable, locally compact, Hausdorff groupoids $G$ and $H$ are equivalent, then the associated full $C^{*}$-algebras are Morita equivalent. This result descends to reduced $C^{*}$-algebras, and also persists for groupoids which are locally Hausdorff (see [20]). The proof of this statement in [20] proceeds by constructing a linking groupoid $L$ from copies of $G, H, Z$ and the opposite space $Z^{\text {op }}$ so that the groupoid $C^{*}$-algebra of $L$ is a linking algebra for a $C^{*}(G)-C^{*}(H)$-imprimitivity bimodule.

Given a Hausdorff ample groupoid $G$ and a commutative unital ring $R$, we consider the convolution $R$-algebra $A_{R}(G)$ of locally constant functions with compact support from $G$ to $R$. We call $A_{R}(G)$ the Steinberg algebra associated to $G$. These algebras were introduced in [22] as a model for discrete inverse semigroup algebras. In the situation where $R=\mathbb{C}, A_{\mathbb{C}}(R)$ is a dense subalgebra of $C^{*}(G)$. Complex Steinberg algebras also include complex Kumjian-Pask algebras [2] and hence complex Leavitt path algebras. Uniqueness theorems and simplicity criteria for complex Steinberg algebras are established in 4 and [6]. These results indicate that the groupoid approach is a good unifying framework for understanding the striking similarities between the theory of graph $C^{*}$ algebras and the theory of Leavitt path algebras, which have attracted a lot of attention in recent years.

In this paper we present further evidence for this viewpoint. First we show that all Leavitt path $R$-algebras can be realised as Steinberg algebras (see example 3.2). Next we show that if $G$ and $H$ are Hausdorff ample groupoids, and if $Z$ is a $G-H$ equivalence,

Date: September 4, 2018.

1991 Mathematics Subject Classification. 16S99 (Primary); 16S10, 22A22 (Secondary).

Key words and phrases. Groupoid; groupoid equivalence; Morita equivalence; linking algebra; Leavitt path algebra.

This research was supported by the Australian Research Council. 
then the linking-groupoid construction of [20] yields another Hausdorff ample groupoid $L$. We then show that the Steinberg algebra $A_{R}(L)$ is, in the appropriate sense, a linking algebra for a surjective Morita context between $A_{R}(G)$ and $A_{R}(H)$, and hence that these two algebras are Morita equivalent.

We conclude by applying our result to the "collapsible subgraph" construction of Crisp and Gow [7]. They identify a specific type of subgraph $T$ of a countable directed graph $E$ and a collapsing process that yields a new graph $F$ with vertices $E^{0} \backslash T^{0}$, and show that $C^{*}(E)$ and $C^{*}(F)$ are Morita equivalent by realising one as a full corner of the other. We show that this is an instance of the Morita-equivalence theorem of [14] using the notion of an abstract transversal of the groupoid of $E$ (see [14, Example 2.7]). We conclude that for arbitrary directed graphs $E$ and commutative unital rings $R$, Crisp and Gow's collapsible subgraph construction yields Morita equivalent Leavitt path $R$-algebras $L_{R}(E)$ and $L_{R}(F)$.

\section{Preliminaries}

A groupoid is a small category in which every morphism has an inverse. Given a groupoid $G$, we write $r(\alpha)$ and $s(\alpha)$ for the range and source of $\alpha \in G$. We call the common image of $r$ and $s$ the unit space of $G$ and denote it $G^{(0)}$. We identify the set of identity morphisms of $G$ with $G^{(0)}$.

An étale groupoid is a groupoid $G$ endowed with a topology so that composition and inversion are continuous, and the source map $s$ is a local homeomorphism. In this case, $r$ is also a local homeomorphism and there is a basis of open bisections; that is, a basis of sets $B \subseteq G$ such that $s$ and $r$ restricted to $B$ are homeomorphisms. We say a groupoid is ample if it has a basis of compact open bisections. Note that a Hausdorff groupoid is ample if and only if it is locally compact, Hausdorff and étale and its unit space is totally disconnected (see [6, Lemma 2.1]). See [16] for more details on étale and ample groupoids.

We use the notational convention that if $A, B$ are subsets of a groupoid $G$, then

$$
A B:=\{\alpha \beta: \alpha \in A, \beta \in B, s(\alpha)=r(\beta)\} .
$$

If $A=\{\alpha\}$, then we write $\alpha B$ for $\{\alpha\} B$. The orbit of a unit $x \in G^{(0)}$ is the set

$$
[x]:=s(x G)=r(G x) \subseteq G^{(0)} .
$$

An (algebraic) isomorphism $\Phi: G \rightarrow H$ of groupoids is a bijection from $G$ to $H$ that carries units to units, preserves the range and source maps and satisfies $\Phi(\alpha \beta)=\Phi(\alpha) \Phi(\beta)$ whenever $\alpha$ and $\beta$ are composable in $G$. Uniqueness of inverses implies that $\Phi\left(\alpha^{-1}\right)=$ $\Phi(\alpha)^{-1}$. If $G$ and $H$ are topological groupoids then an isomorphism $\Phi: G \rightarrow H$ is an algebraic isomorphism that is also a homeomorphism.

The next example demonstrates how groupoids are useful in the study of graph algebras.

Example 2.1. Let $E=\left(E^{0}, E^{1}, r_{E}, s_{E}\right)$ be an arbitrary directed graph 11 We denote the infinite-path space by $E^{\infty}$ and the finite-path space by $E^{*}$. We use the convention that a path $x$ is a sequence of edges $x_{i}$ in which each $s_{E}\left(x_{i}\right)=r_{E}\left(x_{i+1}\right)$ and we write $|x|$ for the length of $x$. A source in $E$ is a vertex $v$ such that $r_{E}^{-1}(v)=\emptyset$, and an infinite receiver is a vertex $v$ such that $r_{E}^{-1}(v)$ is infinite.

\footnotetext{
${ }^{1}$ To avoid confusion, we adopt the convention that an unadorned $r$ or $s$ will always denote the range or source map in a groupoid, and the range and source maps associated to a graph $E$ will always be decorated with a subscript $E$.
} 
The following construction of a groupoid $G_{E}$ from a graph $E$ can be found in [15]. This generalises the construction in [13]. Unlike [13] and [15], we do not require our graphs to be countable. More general versions are described in [9, 12, 18, 26].

Define

$$
X:=E^{\infty} \cup\left\{\mu \in E^{*} \mid s_{E}(\mu) \text { is a source }\right\} \cup\left\{\mu \in E^{*} \mid s_{E}(\mu) \text { is an infinite receiver }\right\} .
$$

Let

$$
G_{E}:=\left\{(\alpha x,|\alpha|-|\beta|, \beta x) \mid \alpha, \beta \in E^{*}, x \in X, s_{E}(\alpha)=s_{E}(\beta)=r_{E}(x)\right\}
$$

We view each $(x, k, y) \in G_{E}$ as a morphism with range $x$ and source $y$. The formulas

$$
(x, k, y)(y, l, z):=(x, k+l, z) \quad \text { and } \quad(x, k, y)^{-1}:=(y,-k, x)
$$

define composition and inverse maps on $G_{E}$ making it a groupoid with

$$
G_{E}^{(0)}=\{(x, 0, x): x \in X\} \text { which we identify with } X .
$$

Next, we describe a topology on $G$. For $\mu \in E^{*}$, the cylinder set $Z(\mu) \subseteq X$ is the set

$$
Z(\mu):=\left\{\mu x \mid x \in X, s_{E}(\mu)=r_{E}(x)\right\} .
$$

For $\mu \in E^{*}$ and a finite $F \subseteq r_{E}^{-1}\left(s_{E}(\mu)\right)$, define

$$
Z(\mu \backslash F):=Z(\mu) \cap\left(\bigcup_{\alpha \in F} Z(\mu \alpha)\right)^{c} .
$$

The sets $Z(\mu \backslash F)$ are a basis of compact open sets for a locally compact, Hausdorff topology on $X=G_{E}^{(0)}$ (see [24, Theorem 2.1]).

For $\mu, \nu \in E^{*}$ with $s_{E}(\mu)=s_{E}(\nu)$, and for a finite $F \subseteq E^{*}$ such that $s_{E}(\mu)=r_{E}(\alpha)$ for all $\alpha \in F$, we define

$$
Z(\mu, \nu):=\left\{(\mu x,|\mu|-|\nu|, \nu x): x \in X, s_{E}(\mu)=r_{E}(x)\right\}
$$

and then

$$
Z((\mu, \nu) \backslash F):=Z(\mu, \nu) \cap\left(\bigcup_{\alpha \in F} Z(\mu \alpha, \nu \alpha)\right)^{c} .
$$

The $Z((\mu, \nu) \backslash F)$ form a basis of compact open sets for a locally compact Hausdorff topology on $G_{E}$ under which it is étale. Hence, $G_{E}$ is a Hausdorff ample groupoid. We will come back to this example in Example 3.2 and again in Section 6.

\section{Steinberg Algebras OVER COMmutative Rings With 1}

Throughout this section, $R$ denotes a commutative unital ring, $\Gamma$ denotes a discrete group, $G$ denotes a Hausdorff ample groupoid, and $c$ denotes a continuous homomorphism from $G$ to $\Gamma$; that is, $c: G \rightarrow \Gamma$ is a continuous groupoid cocycle. The Steinberg algebra $A(G)$ of $G$, introduced in 22 ] 2 is the $R$-algebra of locally constant $R$-valued functions on $G$ with compact support, where addition is pointwise and multiplication is given by convolution

$$
(f * g)(\gamma)=\sum_{\alpha \beta=\gamma} f(\alpha) g(\beta)
$$

It is useful to note that

$$
A_{R}(G)=\operatorname{span}\left\{1_{U}: U \text { is a compact open bisection of } G\right\} \subseteq R^{G},
$$

\footnotetext{
${ }^{2}$ Steinberg's notation is $R G$, but we continue to use the notation of [4, 6].
} 
where $1_{U}$ denotes the characteristic function on $U$ (see [22, Proposition 4.3]). We have

$$
1_{U} * 1_{V}=1_{U V}
$$

for compact open bisections $U$ and $V$ (see [22, Proposition 4.5(3)]).

Lemma 3.1. Suppose that $R$ is a commutative unital ring, $G$ is a Hausdorff ample groupoid and $c: G \rightarrow \Gamma$ is a continuous cocycle. The subsets

$$
A_{R}(G)_{n}:=\left\{f \in A_{R}(G): \operatorname{supp}(f) \subseteq c^{-1}(n)\right\}
$$

for $n \in \Gamma$ form a $\Gamma$-grading of $A_{R}(G)$.

Proof. We must show that:

(1) $A_{R}(G)=\bigoplus_{n \in \Gamma} A_{R}(G)_{n}$ as an $R$-module; and

(2) if $f \in A_{R}(G)_{n}$ and $g \in A_{R}(G)_{m}$ then $f * g \in A_{R}(G)_{n+m}$.

Fix a compact open bisection $U \subseteq G$. For (11), it suffices to show that the indicator function $1_{U}$ belongs to $\bigoplus_{n \in \Gamma} A_{R}(G)_{n}$. For $n \in \Gamma$, let $V_{n}:=U \cap c^{-1}(n)$. Since the $c^{-1}(n)$ are disjoint clopen sets and $U$ is compact open, the $V_{n}$ are disjoint compact open subsets of $U$. Further, since $U$ is compact, only finitely many $V_{n}$ are nonempty, and then $1_{U}=\sum_{V_{n} \neq \emptyset} 1_{V_{n}} \in \bigoplus_{n \in \Gamma} A_{R}(G)_{n}$.

For (2), suppose that $f \in A_{R}(G)_{n}$ and $g \in A_{R}(G)_{m}$. For $\gamma \in G$ we have $(f * g)(\gamma)=$ $\sum_{\alpha \beta=\gamma} f(\alpha) g(\beta)$, and so

$$
\operatorname{supp}(f * g) \subseteq \operatorname{supp}(f) \operatorname{supp}(g) \subseteq c^{-1}(n) c^{-1}(m) \subseteq c^{-1}(n+m) .
$$

Therefore $f * g \in A_{R}(G)_{n+m}$.

Example 3.2. Every Leavitt path algebra is a Steinberg algebra. To see this, let $E$ be an arbitrary directed graph, $G_{E}$ the groupoid of Example 2.1 and $R$ a commutative unital ring. We show that the Leavitt path algebra $L_{R}(E)$ is isomorphic to $A_{R}\left(G_{E}\right)$. It is routine to check that the indicator functions $q_{v}:=1_{Z(v)}, v \in E^{0}$ are mutually orthogonal idempotents, and that the indicator functions $t_{e}:=1_{Z(e, s(e))}$ and $t_{e^{*}}=1_{Z(s(e), e)}$ constitute a Leavitt $E$-family as in [23, Definition 2.4]. So the universal property of $L_{R}(E)$ gives a homomorphism $\pi: L_{R}(E) \rightarrow A_{R}\left(G_{E}\right)$ satisfying $\pi\left(p_{v}\right)=q_{v}, \pi\left(s_{e}\right)=t_{e}$ and $\pi\left(s_{e^{*}}\right)=t_{e^{*}}$. An application of the graded uniqueness theorem [23, Theorem 4.8] shows that this homomorphism is injective. To see that it is surjective, observe that each $1_{Z((\mu, \nu) \backslash F)}=t_{\mu} t_{\nu^{*}}-\sum_{\alpha \in F} t_{\mu \alpha} t_{(\nu \alpha)^{*}}$ belongs to the range of $\pi$. Fix a compact open $U$. This $U$ can be written as a union of basic open sets (because it is open), and therefore as a finite union of basic open sets (because it is compact); say $U=\bigcup_{(\mu, \nu, F) \in \mathcal{F}} Z((\mu, \nu) \backslash F)$. We claim that $U$ can be written as a disjoint union of basic open sets. By the inclusionexclusion principle,

$$
U=\bigsqcup_{\emptyset \neq \mathcal{G} \subseteq \mathcal{F}}\left(\left(\bigcap_{(\mu, \nu, P) \in \mathcal{G}} Z((\mu, \nu) \backslash P)\right) \backslash\left(\bigcup_{(\eta, \zeta, Q) \in \mathcal{F} \backslash \mathcal{G}} Z((\eta, \zeta) \backslash Q)\right)\right)
$$

For any $\mu, \nu, \alpha, \beta \in E^{*}$ with $s(\mu)=s(\nu)$ and $s(\alpha)=s(\beta)$, we have

$$
Z(\mu, \nu) \cap Z(\alpha, \beta)= \begin{cases}Z(\alpha, \beta) & \text { if } \alpha=\mu \tau \text { and } \beta=\nu \tau \\ Z(\mu, \nu) & \text { if } \mu=\alpha \tau \text { and } \nu=\beta \tau \\ \emptyset & \text { otherwise }\end{cases}
$$


and

$$
Z(\mu, \nu) \backslash Z(\alpha, \beta)= \begin{cases}Z((\mu, \nu) \backslash\{\tau\}) & \text { if } \alpha=\mu \tau \text { and } \beta=\nu \tau \\ \emptyset & \text { otherwise. }\end{cases}
$$

Using this, de Morgan's laws and distributivity of intersection and union, it is routine to check that every set of the form $\bigcap_{(\mu, \nu, P) \in \mathcal{G}} Z((\mu, \nu) \backslash P) \backslash\left(\bigcup_{(\eta, \zeta, Q) \in \mathcal{H}} Z((\eta, \zeta), Q)\right)$ with $\mathcal{G}, \mathcal{H}$ finite and $\mathcal{G}$ nonempty can be written as a finite disjoint union of basic open sets. Hence $U$ can be written as a finite disjoint union of basic open sets as claimed. Thus $1_{U}$ is a finite sum of indicator functions of basic open sets, and therefore belongs to the range of $\pi$. That is, $\pi$ is an isomorphism of $L_{R}(E)$ onto $A_{R}\left(G_{E}\right)$ as required.

Remark 3.3. If $\Lambda$ is a row-finite $k$-graph with no sources and $G_{\Lambda}$ is the associated groupoid (see for example [12] and [9]), the [6, Proposition 4.3] shows that $A_{\mathbb{C}}\left(G_{\Lambda}\right)$ is isomorphic to the Kumjian-Pask algebra $\mathrm{KP}_{\mathbb{C}}(\Lambda)$ as defined in [2]. An argument similar to that of the preceding example generalises this to the Kumjian-Pask $R$-algebras associated to a locally convex row-finite $k$-graphs (possibly with sources) as in [5]. That is $\operatorname{KP}_{R}(\Lambda) \cong A_{R}\left(G_{\Lambda}\right)$.

\section{Groupoid equivalence}

In this section, we assume throughout that $G$ is a locally compact Hausdorff groupoid and $X$ is a locally compact Hausdorff space. We say $G$ acts on the left of $X$ if there is a map $r_{X}$ from $X$ onto $G^{(0)}$ and a map $(\gamma, x) \mapsto \gamma \cdot x$ from

$$
G * X:=\left\{(\gamma, x) \in G \times X: s(\gamma)=r_{X}(x)\right\} \text { to } X
$$

such that

(1) if $(\eta, x) \in G * X$ and $(\gamma, \eta)$ is a composable pair in $G$, then $(\gamma \eta, x),(\gamma, \eta \cdot x) \in G * X$ and $\gamma \cdot(\eta \cdot x)=(\gamma \eta) \cdot x$

(2) $r_{X}(x) \cdot x=x$ for all $x \in X$.

We will call $X$ a continuous left $G$-space if $r_{X}$ is an open map and both $r_{X}$ and $(\gamma, x) \mapsto \gamma \cdot x$ are continuous.

The action of $G$ on $X$ is free if $\gamma \cdot x=x$ implies $\gamma=r_{X}(x)$. It is proper if the map from $G * X \rightarrow X \times X$ given by $(\gamma, x) \rightarrow(\gamma \cdot x, x)$ is a proper map in the sense that inverse images of compact sets are compact.

We define right actions similarly, writing $s_{X}$ for the map from $X$ onto $G^{(0)}$, and

$$
X * G:=\left\{(x, \gamma) \in X \times G: s_{X}(x)=r(\gamma)\right\} .
$$

Definition 4.1. Let $G$ and $H$ be locally compact Hausdorff groupoids. A $(G, H)$ equivalence is a locally compact Hausdorff space $Z$ such that

(1) $Z$ is a free and proper left $G$-space;

(2) $Z$ is a free and proper right $H$-space;

(3) the actions of $G$ and $H$ on $Z$ commute;

(4) $r_{Z}$ induces a homeomorphism of $Z / H$ onto $G^{(0)}$;

(5) $s_{Z}$ induces a homeomorphism of $G \backslash Z$ onto $H^{(0)}$.

Suppose that $Z$ is a $(G, H)$-equivalence, and that $y, z, y^{\prime}, z^{\prime} \in Z$ satisfy $s_{Z}(y)=r_{Z}(z)$ and $s_{Z}\left(z^{\prime}\right)=r_{Z}\left(y^{\prime}\right)$. We write ${ }_{G}[y, z] \in G$ and $\left[y^{\prime}, z^{\prime}\right]_{H} \in H$ for the unique elements such that

$$
{ }_{G}[y, z] \cdot z=y \text { and } y^{\prime} \cdot\left[y^{\prime}, z^{\prime}\right]_{H}=z^{\prime} .
$$


Let

$$
Z^{\mathrm{op}}:=\{\bar{z}: z \in Z\}
$$

denote a homeomorphic copy of $Z$. For $z \in Z$, define $r_{Z^{\mathrm{op}}}(\bar{z})=s_{Z}(z) \in H^{(0)}$ and $s_{Z^{\mathrm{op}}}(\bar{z})=r_{Z}(z) \in G^{(0)}$, and for $\eta \in H$ with $s(\eta)=r_{Z^{\mathrm{op}}}(\bar{z})$ and $\gamma \in G$ with $r(\gamma)=s_{Z^{\mathrm{op}}}(\bar{z})$ define

$$
\eta \cdot \bar{z}:=\overline{z \cdot \eta^{-1}} \quad \text { and } \quad \bar{z} \cdot \gamma:=\overline{\gamma^{-1} \cdot z}
$$

With this structure, $Z^{\mathrm{op}}$ is an $(H, G)$-equivalence. See [11, 14, 20] for more information on groupoid actions and equivalences.

Remark 4.2. Note that if $S$ and $T$ are strongly Morita equivalent inverse semigroups as in [21, Definition 2.1], then their respective universal groupoids are equivalent [21, Theorem 4.7].

The linking groupoid. Now suppose that $G$ and $H$ are Hausdorff ample groupoids and let $Z$ be a $(G, H)$-equivalence. We show that $A_{R}(G)$ and $A_{R}(H)$ are Morita equivalent by embedding them as complementary corners of the Steinberg algebra of a linking groupoid $L$ defined below. In the remainder of this section, we verify that the linking groupoid in this situation is also a Hausdorff ample groupoid and then show how $A_{R}(G)$ and $A_{R}(H)$ embed into $A_{R}(L)$.

If $Z$ is a $(G, H)$-equivalence, the linking groupoid of $Z$ is defined in [20, Lemma 2.1] as

$$
L:=G \sqcup Z \sqcup Z^{\mathrm{op}} \sqcup H,
$$

with $r, s: L \rightarrow L^{(0)}:=G^{(0)} \sqcup H^{(0)}$ inherited from the range and source maps on each of $G, H, Z$ and $Z^{\mathrm{op}}$. We write $r$ and $s$ (no subscripts) to denote the range and source maps in $L$. Multiplication $(k, l) \mapsto k l$ in $L$ is given by

- multiplication in $G$ and $H$ when $(k, l)$ is a composable pair in $G$ or $H$;

- $k l=k \cdot l$ when $(k, l) \in Z * H \sqcup G * Z \sqcup H * Z^{\mathrm{op}} \sqcup Z^{\mathrm{op}} * G$; and

- $k l={ }_{G}[k, h]$ if $k \in Z$ and $l=\bar{h} \in Z^{\mathrm{op}}$, and $k l=[h, l]_{H}$ if $l \in Z$ and $k=\bar{h} \in Z^{\text {op }}$.

The inverse map is the usual inverse map in each of $G$ and $H$ and is given by $z \mapsto \bar{z}$ on $Z$ and $\bar{z} \mapsto z$ in $Z^{\mathrm{op}}$. Both $G$ and $H$ are clopen in $L$ by construction.

Lemma 4.3. Let $G$ and $H$ be Hausdorff ample groupoids. Suppose that $Z$ is a $(G, H)$ equivalence and $L$ is the linking groupoid of $Z$. Then $L$ is a Hausdorff ample groupoid.

Proof. Lemma 2.1 of [20] implies that $L$ is locally compact and Hausdorff. It suffices to show that $L$ is étale with totally disconnected unit space. We have $L^{(0)}=G^{(0)} \sqcup H^{(0)}$ which is totally disconnected because $G^{(0)}$ and $H^{(0)}$ are, so it remains to show that $L$ is étale 3

We suppose that $r$ is not a local homeomorphism, and seek a contradiction. Then there exists $z \in L$ such that $r$ fails to be injective on every neighbourhood of $z$. Because $G$ and $H$ are étale, $z$ is either in $Z$ or $Z^{o p}$. Without loss of generality, assume $z \in Z$; the case for $Z^{o p}$ is symmetric. By choosing a neighbourhood base $\left\{U_{\alpha}\right\}$ at $z$ inside of $Z$, we can find a net $\left\{\left(x_{\alpha}, y_{\alpha}\right)\right\}$ where each $x_{\alpha}, y_{\alpha} \in U_{\alpha}$ such that:

(1) $x_{\alpha}, y_{\alpha} \rightarrow z$

\footnotetext{
${ }^{3}$ If $G$ and $H$ were second-countable, then $L$ would be as well, and then we could deduce from [17, Lemma I.2.7 and Proposition I.2.8] that $L$ is étale by observing that $L^{(0)}$ is open in $L$ (because each of $G^{(0)}$ and $H^{(0)}$ is open), and the Haar system on $L$ induced from those on $G$ and $H$ consists of counting measures because the systems on $G$ and $H$ have this property.
} 
(2) $x_{\alpha} \neq y_{\alpha}$ for all $n$;

(3) $r\left(x_{\alpha}\right)=r\left(y_{\alpha}\right)$ for all $n$.

Since $G$ is étale, $G^{(0)}$ is open in $L$ and so we can assume that $r\left(x_{\alpha}\right) \in G^{(0)}$ for all $\alpha$. For each $\alpha$, let $\gamma_{\alpha}:=\left[x_{\alpha}, y_{\alpha}\right]_{H}$, so that $x_{\alpha} \cdot \gamma_{\alpha}=y_{\alpha}$ for all $\alpha$. Note that $r\left(\gamma_{\alpha}\right)=s\left(x_{\alpha}\right)$. Proposition 1.15 of [25] applied to the open map $r: H \rightarrow H^{(0)}$ implies that, by passing to a subnet, we may assume that $\gamma_{\alpha} \rightarrow \gamma \in H$. So the continuity of the action gives

$$
z \cdot \gamma=\lim x_{\alpha} \cdot \gamma_{\alpha}=\lim y_{\alpha}=z .
$$

Since $H$ acts freely on $Z$, this forces $\gamma=s(z)$. Since $H^{(0)}$ is open in $H$, we have $\gamma_{\alpha} \in H^{(0)}$ eventually. Hence $x_{\alpha}=y_{\alpha}$ eventually, contradicting (2).

Following [20, page 108], for each $F \in A_{R}(L)$, define $F_{11}=\left.F\right|_{G}, F_{12}=\left.F\right|_{Z}, F_{21}=$ $\left.F\right|_{Z^{\text {op }}}$ and $F_{22}=\left.F\right|_{H}$. We may view each $F_{i j}$ as an element of $A_{R}(L)$. We express the decomposition $F=\sum_{i, j} F_{i j}$ by writing

$$
F=\left(\begin{array}{ll}
F_{11} & F_{12} \\
F_{21} & F_{22}
\end{array}\right)
$$

It is straightforward to check that convolution in $A_{R}(L)$ is given by matrix multiplication for functions written in this form. Using this notation, we see that the inclusion maps

$$
f \mapsto\left(\begin{array}{cc}
f & 0 \\
0 & 0
\end{array}\right) \text { and } g \mapsto\left(\begin{array}{cc}
0 & 0 \\
0 & g
\end{array}\right)
$$

define injective homomorphisms $A_{R}(G) \hookrightarrow A_{R}(L)$ and $A_{R}(H) \hookrightarrow A_{R}(L)$. We denote the images of these maps by $i\left(A_{R}(G)\right)$ and $i\left(A_{R}(H)\right)$. So

$$
i\left(A_{R}(G)\right) \cong A_{R}(G) \text { and } i\left(A_{R}(H)\right) \cong A_{R}(G) .
$$

\section{MAIN RESULT}

We now have the machinery we need to show that equivalent groupoids give rise to Morita equivalent Steinberg algebras. First, we give the definition of Morita equivalent rings. Let $A$ and $B$ be rings, $M$ an $A-B$ bimodule, $N$ a $B-A$ bimodule, and

$$
\psi: M \otimes_{B} N \rightarrow A \text { and } \phi: N \otimes_{A} M \rightarrow B
$$

bimodule homomorphisms such that

$$
n^{\prime} \cdot \psi(m \otimes n)=\phi\left(n^{\prime} \otimes m\right) \cdot n \text { and } m^{\prime} \cdot \phi(n \otimes m)=\psi\left(m^{\prime} \otimes n\right) \cdot m
$$

for $n, n^{\prime} \in N$ and $m, m^{\prime} \in M$. Then $(A, B, M, N, \psi, \phi)$ is a Morita context between $A$ and $B$; it is called surjective if $\psi$ and $\phi$ are surjective and in this case we say $A$ and $B$ are Morita equivalent. (See [10, page 41].)

Theorem 5.1. Let $G$ and $H$ be Hausdorff ample groupoids. Suppose that $Z$ is a $(G, H)$ equivalence with linking groupoid $L$. Let $i$ denote the inclusion maps from $A_{R}(G)$ and $A_{R}(H)$ into $A_{R}(L)$. Define

$$
M:=\left\{f \in A_{R}(L) \mid \operatorname{supp} f \subseteq Z\right\} \quad \text { and } \quad N:=\left\{f \in A_{R}(L) \mid \operatorname{supp} f \subseteq Z^{\mathrm{op}}\right\},
$$

and let $A_{R}(G)$ and $A_{R}(H)$ act on the right and left of $M$ and on the left and right of $N$ by $a \cdot f=i(a) * f$ and $f \cdot a=f * i(a)$. Then there are bimodule homomorphisms

$$
\psi: M \otimes_{i\left(A_{R}(H)\right)} N \rightarrow A_{R}(G) \quad \text { and } \quad \phi: N \otimes_{i\left(A_{R}(G)\right)} M \rightarrow A_{R}(H)
$$


determined by

$$
i(\psi(f \otimes g))=f * g \quad \text { and } \quad i(\psi(g \otimes f))=g * f .
$$

The tuple $\left(A_{R}(G), A_{R}(H), M, N, \psi, \phi\right)$ is a surjective Morita context, and so $A_{R}(G)$ and $A_{R}(H)$ are Morita equivalent.

Proof. That $M$ is an $A_{R}(G)-A_{R}(H)$ bimodule and $N$ is an $A_{R}(H)-A_{R}(G)$ bimodule is clear. The given formulas for $\phi$ and $\psi$ are well-defined on the balanced tensor products because, for example,

$$
f *(a \cdot g)=f *(i(a) * g)=(f * i(a)) * g=(f \cdot a) * g .
$$

The maps $\psi$ and $\phi$ are module homomorphisms by linearity of convolution. The formula (5.1) follows from associativity of convolution in $A_{R}(L)$.

To see that $\psi$ is surjective, it suffices to fix a compact open bisection $U \subseteq G$ and show that $i\left(1_{U}\right)$ is in the image of $\psi$. For each $x \in r(U)$, choose $z_{x} \in Z$ such that $r\left(z_{x}\right)=x$. Since $L$ is étale and $Z$ is topologically disjoint from $G$, each $z_{x}$ has a neighbourhood $U_{x} \subseteq Z$ which is a bisection of $L$. Since $G^{(0)}$ is locally compact, Hausdorff and totally disconnected, each $x$ has a compact open neighbourhood $W_{x}$ contained in $r(U) \cap r\left(U_{x}\right)$, and so by replacing each $U_{x}$ with $U_{x} \cap r^{-1}\left(W_{x}\right)$, we can assume that each $U_{x}$ is compact open with $r\left(U_{x}\right) \subseteq r(U)$. Since $r(U)$ is compact, there is a finite set $\left\{x_{1}, \ldots, x_{n}\right\} \subseteq r(U)$ such that $\bigcup_{i} r\left(U_{x_{i}}\right)=r(U)$. Let $V_{1}=U_{x_{1}}$ and iteratively define $V_{i}=U_{x_{i}} \backslash r^{-1}\left(\bigcup_{j<i} r\left(U_{x_{j}}\right)\right)$. Then the $V_{i}$ are compact open subsets of $Z$ on which $r$ and $s$ are bijective, and $r(U)$ is the disjoint union of the $r\left(V_{i}\right)$. Therefore, writing $V_{i}^{\text {op }}$ for $\left\{\bar{z}: z \in V_{i}\right\} \subseteq Z^{\mathrm{op}}$, we have

$$
\left(\begin{array}{cc}
1_{U} & 0 \\
0 & 0
\end{array}\right)=\sum_{i}\left(\begin{array}{cc}
0 & 1_{V_{i}} \\
0 & 0
\end{array}\right)\left(\begin{array}{cc}
0 & 0 \\
1_{V_{i}^{\mathrm{po}}} & 0
\end{array}\right) .
$$

Thus $1_{U}=\psi\left(\sum_{i} 1_{V_{i}} \otimes 1_{V_{i}}^{\text {op }}\right)$, and so $\psi$ is surjective. A similar argument shows that $\phi$ is surjective.

It follows that $\left(A_{R}(G), A_{R}(H), M, N, \psi, \phi\right)$ is a surjective Morita context, and so $A_{R}(G)$ and $A_{R}(H)$ are Morita equivalent.

\section{Applications to graph algebras}

Our aim is to apply our main result to graph algebras. First we consider a useful class of examples of groupoid equivalences - those arising from abstract transversals of groupoids. Suppose that $G$ is a subgroupoid 4 of $H$ and let $Z:=G^{(0)} H$. It is straightforward to check that $Z$ is a free and proper left $G$-space and a free and proper right $H$ space where $r_{Z}$ and $s_{Z}$ are the range and source from $H$ restricted to $Z$ and the action is by multiplication in $H$. Because groupoid multiplication is associative, the actions of $G$ and $H$ commute. However, $Z$ may not satisfy the surjectivity hypothesis of Definition 4.1 (5) required in a groupoid equivalence. The following lemma is a straightforward application of [14, Example 2.7]; we give a short proof because the construction is fundamental to our application of groupoid equivalence to graph algebras.

Lemma 6.1. Suppose $H$ is an étale groupoid and $X \subseteq H^{(0)}$ is a clopen subset that meets each orbit in $H$. Then $G:=X H X$ is a clopen subgroupoid of $H$, and $Z:=X H$ is a $(G, H)$-equivalence.

\footnotetext{
${ }^{4}$ By subgroupoid we mean a subset that is itself a groupoid.
} 
Proof. The set $X H X=r^{-1}(X) \cap s^{-1}(X)$ is clopen because $r$ and $s$ are continuous, and it is clearly a subgroupoid. Similarly, $Z$ is a clopen subset of $H$, and so the open subsets of $Z$ are the subsets of $Z$ which are open in $H$. Since $H$ is étale, $r$ and $s$ are open maps and so $r_{Z}$ and $s_{Z}$ (which are $r$ and $s$ restricted to $Z$ ) are also open maps. The map $r_{Z}: Z \rightarrow X$ is surjective by definition. To see that $s_{Z}: Z \rightarrow H^{(0)}$ is surjective, fix $u \in H^{(0)}$. By hypothesis, $[u] \cap X \neq \emptyset$, so there exists $\alpha \in H$ such that $r(\alpha) \in X$ and $u=s(\alpha)$. So $\alpha \in Z$ and $u=s(\alpha) \in s_{Z}(Z)$.

We prove that $\tilde{s}: G \backslash Z \rightarrow H^{(0)}$ is a homeomorphism; the argument that $\tilde{r}$ is a homeomorphism is similar. Clearly, $\tilde{s}$ is a surjection. If $\tilde{s}([\alpha])=\tilde{s}([\beta])$, then $s(\alpha)=s(\beta)$, and so $\alpha \beta^{-1} \in X H X=G$ and satisfies $\left(\alpha \beta^{-1}\right) \cdot \beta=\alpha$. So $[\alpha]=[\beta]$, and $\tilde{s}$ is injective.

To see that $\tilde{s}$ is continuous, suppose $U \subseteq H^{(0)}$ is open. Then $H U$ is open because $s$ is continuous, and then $Z U=H U \cap Z$ is open in $Z$. Thus $\tilde{s}^{-1}(U)=G \backslash(Z U)$ is open by definition of the quotient topology.

Finally, if $W \subseteq G \backslash Z$ is open, then $W=G \backslash W^{\prime}$ for some open $W^{\prime} \subseteq Z$. Since $Z$ is open in $H$, so is $W^{\prime}$ and then $\tilde{s}(W)=s\left(W^{\prime}\right)$ is open because $s$ is open.

Given a graph $E$, Crisp and Gow identify a type of subgraph $T$ which can be "collapsed" to yield a new graph $F$ whose $C^{*}$-algebra is Morita equivalent to that of $E$ [7]. We will demonstrate that $G_{E}$ and $G_{F}$ are equivalent groupoids. Bates and Pask's "outsplitting" move described in [3, Theorem 4.5 and Corollary 5.4] is a special case of the CrispGow construction (see [7, Example iii]), as are Sørensen's moves (S) and (R) (see [19, Propositions 3.1 and 3.2]). So our result implies that applications of these moves yield Morita equivalent Leavitt path algebras regardless of the base ring.

When $E$ is countable, our statement of the next proposition corresponds exactly to the construction of [7, Theorem 3.1] modulo the difference in edge-direction conventions. First, we need a few more graph preliminaries. Suppose $E$ is a directed graph. For $v \in E^{0}$ and $S \subseteq E^{0}$, we write $v \geq S$ if $S E^{*} v \neq \emptyset$. We define the pointed groupoid with respect to $S$ to be the subgroupoid of $G_{E}$ consisting of groupoid elements $(\alpha x,|\alpha|-|\beta|, \beta x)$ such that $r_{E}(\alpha), r_{E}(\beta) \in S$. We define

$$
E_{\text {sing }}^{0}:=\left\{v \in E^{0}: r_{E}^{-1}(v) \text { is either empty or infinite }\right\} .
$$

For $n \in \mathbb{N}$ we define a map $\sigma^{n}:\left\{x \in E^{*} \cup E^{\infty}:|x| \geq n\right\} \rightarrow E^{*} \cup E^{\infty}$ by $\sigma^{n}(\alpha y)=y$ for all $\alpha \in E^{n}$ (paths of length $n$ ) and $y \in E^{*} \cup E^{\infty}$. Notice that $G_{E}^{(0)}$ is invariant under $\sigma^{n}$. Finally, we say an acyclic path $x \in E^{\infty}$ is a head if each $r_{E}\left(x_{i}\right)$ receives only $x_{i}$ and each $s_{E}\left(x_{i}\right)$ emits only $x_{i}$.

Proposition 6.2. Let $E$ be a directed graph with no heads and suppose that $F^{0} \subseteq E^{0}$ satisfies $E_{\text {sing }}^{0} \subseteq F^{0}$. Suppose also that the subgraph $T$ of $E$ defined by $T^{0}:=E^{0} \backslash F^{0}$ and

$$
T^{1}:=\left\{e \in E^{1}: r_{E}(e), s_{E}(e) \in T^{0}\right\}
$$

is acyclic and that each of the following are satisfied:

(T1) each vertex in $F^{0}$ is the range of at most one $y \in E^{\infty}$ such that $s_{E}\left(y_{i}\right) \in T^{0}$ for all $i \geq 1$;

and for each $x \in T^{\infty}$,

(T2) $r_{E}(x) \geq F^{0}$

(T3) $\left|s_{E}^{-1}\left(r_{E}\left(x_{i}\right)\right)\right|=1$ for all $i$; and

(T4) whenever $s_{E}(e)=r_{E}(x)$, we have $\left|r_{E}^{-1}\left(r_{E}(e)\right)\right|<\infty$. 
Let $F$ be the graph with vertex set $F^{0}$ and one edge $e_{\beta}$ for each path $\beta \in E^{*} \backslash E^{0}$ with $s_{E}(\beta), r_{E}(\beta) \in F^{0}$ and $r_{E}\left(\beta_{i}\right) \in T^{0}$ for $1 \leq i<|\beta|$ such that $s_{F}\left(e_{\beta}\right)=s_{E}(\beta)$ and $r_{F}\left(e_{\beta}\right)=r_{E}(\beta)$. Let $G \subseteq G_{E}$ denote the pointed groupoid with respect to $F^{0}$. Then

(1) $G$ and $G_{E}$ are equivalent groupoids and

(2) $G$ is isomorphic to $G_{F}$.

Remark 6.3. We will be using [7, Lemma 3.3], which says that if a graph $E$ has no heads, satisfies (T1), (T2) and (T3), and $T$ and $F$ are as above, then $F^{0} \geq v$ for all $v \in T^{0}$. Note that this Lemma also implies that $r_{E}^{-1}(v)=\emptyset$ if and only if $r_{F}^{-1}(v)=\emptyset$.

Proof. To prove (11), we will apply Lemma 6.1 with $X=G^{(0)}=F^{0} E^{\infty}$. First notice that

$$
G^{(0)}=\bigcup_{v \in F^{0}} Z(v)=G_{E}^{(0)} \backslash\left(\bigcup_{w \in T^{0}} Z(w)\right)
$$

Since each $Z(v)$ is open, we deduce that $G^{(0)}$ is clopen in $G_{E}^{(0)}$. Now consider $x \in G_{E}^{(0)} \backslash G^{(0)}$. We must show that $[x] \cap G^{(0)} \neq \emptyset$. Since $x \notin G^{(0)}, r_{E}(x) \in T^{0}$. We consider 2 cases. For the first case, suppose that $\sigma^{n}(x) \in T^{\infty}$ for some $n$. Then (T2) implies that there exists $\mu \in E^{*}$ such that $s_{E}(\mu)=r_{E}\left(x_{n+1}\right)$ and $r_{E}(\mu) \in F^{0}$. So $\mu\left(\sigma^{n}(x)\right) \in[x] \cap G^{(0)}$. For the second case, suppose that $\sigma^{n}(x) \notin T^{\infty}$ for all $n$. Since $E_{\text {sing }}^{0} \subseteq F^{0}$, there exists $n$ such that $s_{E}\left(x_{n}\right) \in F^{0}$. Hence $\sigma^{n}(x) \in[x] \cap G^{(0)}$. Now Lemma 6.1 implies that $X G_{E}$ is a $\left(G, G_{E}\right)$-equivalence.

To prove (2), we first define a map $\phi: G_{F}^{(0)} \rightarrow G^{(0)}$, which will take a little preparation. By construction, $F^{1}$ is a subset of $E^{*}$; we write $\phi_{\text {fin }}: F^{1} \rightarrow E^{*}$ for the inclusion map. Since $\phi_{\text {fin }}$ preserves ranges and sources, we can extend $\phi_{\text {fin }}$ to an injection from $F^{*}$ to $E^{*}$ by

$$
\phi_{\text {fin }}(\mu)=\phi_{\text {fin }}\left(\mu_{1}\right) \phi_{\text {fin }}\left(\mu_{2}\right) \ldots \phi_{\text {fin }}\left(\mu_{|\mu|}\right) .
$$

Again by construction of $F$, we have

$$
\phi_{\text {fin }}\left(F^{*}\right)=\left\{\mu \in E^{*}: r_{E}(\mu), s_{E}(\mu) \in F^{0}\right\} .
$$

We claim that if $v \in F^{0}$ satisfies $\left|r_{F}^{-1}(v)\right|=\infty$ but $\left|r_{E}^{-1}(v)\right|<\infty$, then there is a unique infinite path $y_{v} \in T^{\infty}$ with $r_{E}\left(y_{v}\right)=v$. Indeed, the set

$$
B_{v}:=\left\{\beta \in E^{*} \backslash E^{0} \mid r_{E}(\beta)=v, s_{E}(\beta) \in F^{0} \text { and } r_{E}\left(\beta_{i}\right) \in T^{0} \text { for } 1 \leq i \leq|\beta|\right\}
$$

is infinite, and so [7, Lemma 3.4(d)] gives such a $y_{v}$. That there is a unique such path follows from (T1).

Define $\phi: G_{F}^{(0)} \rightarrow G^{(0)}$ by

$$
\phi(x)= \begin{cases}\phi_{\text {fin }}\left(x_{1}\right) \phi_{\text {fin }}\left(x_{2}\right) \ldots & \text { if } x \in F^{\infty} ; \\ \phi_{\text {fin }}(x) & \text { if } x \in F^{*} \text { and } s_{F}(x) \in E_{\text {sing }}^{0} ; \text { and } \\ \phi_{\text {fin }}(x) y_{s_{F}(x)} & \text { if } x \in F^{*},\left|r_{F}^{-1}\left(s_{F}(x)\right)\right|=\infty, \text { and } 0<\left|r_{E}^{-1}\left(s_{F}(x)\right)\right|<\infty .\end{cases}
$$

To see that this defines $\phi$ on all $G_{F}^{(0)}$ observe that if $x \in G_{F}^{(0)}$ belongs to $F^{*}$ and $s_{F}(x) \notin$ $E_{\text {sing }}^{0}$, then we have $s_{F}(x) \in F_{\text {sing }}^{0} \backslash E_{\text {sing }}^{0}$, and since $r_{E}^{-1}(v)=\emptyset$ if and only if $r_{F}^{-1}(v)=\emptyset$, we deduce that $\left|r_{F}^{-1}\left(s_{F}(x)\right)\right|=\infty$ and $0<\left|r_{E}^{-1}\left(s_{F}(x)\right)\right|<\infty$.

Since $\phi_{\text {fin }}$ is injective, $\phi$ is also injective. We have

$$
\begin{aligned}
& \phi\left(F^{\infty}\right)=\left\{x \in F^{0} E^{\infty} \mid s_{E}\left(x_{n}\right) \in F^{0} \text { for infinitely many } n\right\} \text { and } \\
& \phi\left(\left\{\mu \in F^{*}: s_{F}(x) \in E_{\text {sing }}^{0}\right\}\right)=\left\{\mu \in F^{0} E^{*}: s_{E}(\mu) \in E_{\text {sing }}^{0}\right\}
\end{aligned}
$$


because $E_{\text {sing }}^{0} \subseteq F^{0}$. The complement of these two sets in $G^{(0)}$ is

$$
\begin{aligned}
\left\{x \in F^{0} E^{\infty}\right. & \left.\mid s_{E}\left(x_{i}\right) \notin F^{0} \text { eventually }\right\} \\
& =\left\{x \in F^{0} E^{\infty} \mid s_{E}\left(x_{i}\right) \in T^{0} \text { eventually }\right\} \\
& =\left\{\mu y \mid \mu \in F^{0} E^{*} F^{0}, y \in s_{E}(\mu) E^{\infty}, \sigma^{1}(y) \in T^{\infty}\right\} .
\end{aligned}
$$

Let $\mu y$ be an element of the set (6.2). To see that $\phi$ is surjective, it suffices to show that $\left|r_{F}^{-1}\left(r_{E}(y)\right)\right|=\infty$, and $0<\left|r_{E}^{-1}\left(r_{E}(y)\right)\right|<\infty$. For then $\phi\left(\phi_{\text {fin }}^{-1}(\mu)\right)=\mu y$. Condition $(\mathrm{T} 4)$ applied to $e=y_{1}$ implies that $r_{E}\left(y_{1}\right)$ is not an infinite receiver in $E$. We must now show that $r_{F}^{-1}\left(r_{E}\left(y_{1}\right)\right)$ is infinite. Since $T$ is acyclic, $y$ has no repeating edges or vertices. Lemma 3.3 of [7] yields a path $\mu^{1} \in E^{*}$ with $r_{E}\left(\mu^{1}\right)=s_{E}\left(y_{1}\right)$ and $s_{E}\left(\mu^{1}\right)=v_{1} \in F^{0}$. Since $s_{E}\left(\mu^{1}\right) \in F^{0}$, (T3) implies that there exists $m_{1}<\left|\mu^{1}\right|$ such that $y_{j} \notin\left\{\mu_{m_{1}}^{1}, \ldots, \mu_{\left|\mu^{1}\right|}^{1}\right\}$ for all $j$.

Repeating this process for each $n \in \mathbb{N}$, we obtain distinct paths $\mu^{n}$ such that $r_{E}\left(\mu^{n}\right)=$ $s_{E}\left(y_{k_{n}}\right)$ where $k_{n}=\sum_{i=1}^{n}\left(m_{i}+2\right)$ and $s_{E}\left(\mu^{n}\right) \in F^{0}$. Now $y_{1} \ldots y_{k_{n}} \mu^{n} \in r_{F}^{-1}\left(r_{E}(y)\right)$ for all $n$, and these are distinct elements of $F^{1}$, so that $r_{F}^{-1}(r(y))$ is infinite as required. Therefore, $\phi$ is surjective. Notice that $\phi$ also preserves concatenation of paths.

Next we show that $\phi$ is a homeomorphism. It takes cylinder sets $Z(\mu)$ in $G_{F}^{(0)}$ onto cylinder sets $Z\left(\phi_{\text {fin }}(\mu)\right)$ of $G^{(0)}$, and since it is bijective, it is therefore open.

To see that $\phi$ is continuous, suppose $x^{n} \rightarrow x$ in $G_{F}^{(0)}$. We consider the three possibilities for $x$. First, if $x \in F^{\infty}$, then the collection $\left\{Z\left(x_{1}\right), Z\left(x_{1} x_{2}\right), \ldots\right\}$ is a neighbourhood base at $x$ and the collection

$$
\left\{\phi\left(Z\left(x_{1}\right)\right), \phi\left(Z\left(x_{1} x_{2}\right)\right), \ldots\right\}=\left\{Z\left(\phi\left(x_{1}\right)\right), Z\left(\phi\left(x_{1} x_{2}\right)\right), \ldots\right\}
$$

is a neighbourhood base for $\phi(x)$. So $\phi\left(x^{n}\right)$ converges to $\phi(x)$.

Second, if $x \in F^{*}$ and $s_{F}(x)$ is a source, then $\{x\}$ is open in $G_{F}^{(0)}$ and hence $x^{n}=x$ eventually. Therefore $\phi\left(x^{n}\right)=\phi(x)$ eventually and hence $\phi\left(x^{n}\right)$ converges to $\phi(x)$.

Finally, suppose $x \in F^{*}$ and $s(x)$ is an infinite receiver. If $x^{n}$ is eventually constant then $\phi\left(x^{n}\right)$ converges to $\phi(x)$ as above. So suppose otherwise. Since $x^{n} \in Z(x)$ eventually, we may assume that each $x^{n}=x z^{n}$ where $z^{n} \in G_{F}^{(0)}$. Also, we have that $\phi(x)=\phi_{\text {fin }}(x) y_{s_{E}(x)}$. Let $B:=Z\left(\phi_{\text {fin }}(x) y_{1} \ldots y_{m}\right)$ be a basis element containing $\phi(x)$. Since open sets containing $x$ include sets of the form

$$
Z(x) \cap\left(\bigcup_{e \in G} Z(x e)\right)^{c}
$$

for finite $G \subseteq r_{F}^{-1}\left(s_{F}(x)\right)$, we may assume that $z_{1}^{n} \neq z_{1}^{m}$ for $n \neq m$; that is, the first edges of the paths $z^{n}$ are distinct. Condition (T4) implies that $s_{F}(x)$ is not an infinite receiver in $E$, so we may also assume that $\phi\left(z_{1}^{n}\right) \in E^{*} \backslash E^{1}$ for each $n$. So the $\phi\left(z_{1}^{n}\right)$ are paths in $E$ with range and source in $F^{0}$ but all other vertices in $T^{0}$. We claim that the distinct paths $\phi\left(z^{n}\right)$ eventually belong to $Z\left(y_{1} y_{2} \ldots y_{m}\right)$. Note that [7, Lemma 3.3] and (T3) imply that $\left|B_{s_{E}\left(y_{1}\right)}\right|$ is infinite. Further, for any $e \in r_{E}^{-1}\left(s_{F}(x)\right) \backslash\left\{y_{1}\right\}$ we have $\left|B_{s_{E}(e)}\right|<\infty$; for otherwise [7, Lemma 3.4(d)] yields an infinite path that violates (T1). Hence $\phi\left(z^{n}\right) \in Z\left(y_{1}\right)$ eventually. Similarly, $\left|B_{s_{E}\left(y_{2}\right)}\right|$ is infinite and for any $e \in E^{1}$ with $r_{E}(e)=r_{F}\left(y_{2}\right)$ we have $\left|B_{s_{E}(e)}\right|<\infty$ so $\phi\left(z^{n}\right) \in Z\left(y_{1} y_{2}\right)$ for large $n$. Proceeding in this way, we deduce that for any $m$ we have $\phi\left(z^{n}\right) \in Z\left(y_{1} \ldots y_{m}\right)$ for large $n$ as claimed. So $\phi\left(x\left(z_{n}\right)\right) \in B$ for large $n$. Thus, $\phi$ is continuous and hence $\phi$ is a homeomorphism. 
Define $\Phi: G_{F} \rightarrow G$ by

$$
\Phi(\mu x,|\mu|-|\nu|, \nu x)=\left(\phi(\mu x),\left|\phi_{\text {fin }}(\mu)\right|-\left|\phi_{\text {fin }}(\nu)\right|, \phi(\nu x)\right) .
$$

Since $\phi$ preserves concatenation of paths, $\Phi$ is a groupoid homomorphism and it is straightforward to show that $\Phi$ is bijective using that $\phi$ is bijective. We have

$$
\Phi(Z(\mu, \nu))=Z\left(\phi_{\text {fin }}(\mu), \phi_{\text {fin }}(\nu)\right)
$$

for all $\mu, \nu \in F^{*}$. So $\Phi$ takes basic open sets in $G_{F}$ to basic open sets in $G$, and hence $\Phi$ is open.

To see that $\Phi$ is continuous, suppose $\gamma_{n}$ converges to $\gamma=(\mu x, k, \nu x) \in G_{F}$. So for a basis element

$$
B:=Z\left(\mu x_{1} \ldots x_{m}, \nu x_{1} \ldots x_{m}\right) \cap\left(\bigcup_{\alpha \in F} Z\left(\mu x_{1} \ldots x_{n} \alpha, \nu x_{1} \ldots x_{m} \alpha\right)\right)^{c}
$$

containing $\gamma \in G_{F}$, we eventually have $\gamma_{n} \in B$. So for large $n$, the element $\gamma_{n}$ has the form

$$
\gamma_{n}=\left(\mu x_{1} \ldots x_{m} y^{n}, k, \nu x_{1} \ldots x_{m} y^{n}\right) \text { for } y^{n} \in G_{F}^{(0)} .
$$

Thus eventually we have

$$
\Phi\left(\gamma_{n}\right)=\left(\phi\left(\left(\mu x_{1} \ldots x_{m} y^{n}\right),\left|\phi_{\text {fin }}(\mu)\right|-\left|\phi_{\text {fin }}(\nu)\right|, \phi\left(\nu x_{1} \ldots x_{m} y^{n}\right)\right),\right.
$$

which converges to $\left(\phi(\mu x),\left|\phi_{\text {fin }}(\mu)\right|-\left|\phi_{\text {fin }}(\nu)\right|, \phi(\nu x)\right)=\Phi(\gamma)$.

Corollary 6.4. Suppose $E$ and $F$ are as in Proposition 6.2 and $R$ is a commutative unital ring. Then

(1) $L_{R}(E)$ is Morita equivalent to $L_{R}(F)$; and

(2) If $E$ is countable, then $C^{*}(E)$ is Morita equivalent to $C^{*}(F)$.

Proof. Proposition 6.2 implies that $G_{E}$ and $G_{F}$ are equivalent groupoids.

Now for (11), Theorem 5.1 implies that $A_{R}\left(G_{E}\right)$ and $A_{R}\left(G_{F}\right)$ are Morita equivalent, and the result follows from Example 3.2 .

For (2), observe that since $E$ is countable, $G_{E}$ is second countable and hence $C^{*}\left(G_{E}\right)$ is Morita equivalent to $C^{*}\left(G_{F}\right)$ by [14, Theorem 2.8]. We have $C^{*}\left(G_{E}\right) \cong C^{*}(E)$ and $C^{*}\left(G_{F}\right) \cong C^{*}(F)$ by [15, Corollary 3.9], and the result follows.

Remark 6.5. Corollary 6.4(11) generalises [1, Proposition 1.11]. Our proof of Corollary 6.4(2) provides an alternative proof of [7, Theorem 3.1].

Remark 6.6. Sørensen's move (I) of [19, Theorem 3.5] is a special case of Bates and Pask's construction "insplitting" in [3, Theorem 5.3]; a Leavitt path algebra version of this is proved in [1, Proposition 1.14]. In this setting, the corresponding algebras are actually stably isomorphic. Both [19, Theorem 3.5] and [1, Proposition 1.14] can be proved via Steinberg algebras by showing that the corresponding groupoids are isomorphic. This was done in the row-finite case by Drinen in [8, Proposition 6.1.3].

\section{REFERENCES}

[1] G. Abrams, A. Louly, E. Pardo and C. Smith, Flow invariants in the classification of Leavitt path algebras, J. Algebra 333 (2011), 202-231.

[2] G. Aranda Pino, J. Clark, A. an Huef and I. Raeburn, Kumjian-Pask algebras of higher-rank graphs, Trans. Amer. Math. Soc. 365 (2013), 3613-3641. 
[3] T. Bates and D. Pask, Flow equivalence of graph algebras, Ergodic Theory Dynam. Systems 24 (2004), 367-382.

[4] J.H. Brown, L.O. Clark, C. Farthing and A. Sims, Simplicity of algebras associated to étale groupoids, Semigroup Forum, to appear.

[5] L.O. Clark, C. Flynn and A. an Huef, Kumjian-Pask algebras of locally convex higher-rank graphs, J. Algebra 399 (2014), 445-474.

[6] L.O. Clark, C. Farthing, A. Sims and M. Tomforde, A groupoid generalisation of Leavitt path algebras, preprint (arXiv:1110.6198 [math.RA]).

[7] T. Crisp and D. Gow, Contractible subgraphs and Morita equivalence of graph $C^{*}$-algebras, Proc. Amer. Math. Soc. 134 (2006), 2003-2013.

[8] D. Drinen, Flow equivalence and graph groupoid isomorphism, PhD Thesis (1999), Arizona State University, USA.

[9] C. Farthing, P. S. Muhly, and T. Yeend, Higher-rank graph $C^{*}$-algebras: an inverse semigroup and groupoid approach, Semigroup Forum 71 (2005), 159-187.

[10] J.L. García and J.J. Simón, Morita equivalence for idempotent rings, J. Pure Appl. Algebra 76 (1991), 39-56.

[11] G. Goehle, Groupoid Crossed Prodcuts, PhD Thesis (2009), Dartmouth College, USA.

[12] A. Kumjian and D. Pask, Higher rank graph $C^{*}$-algebras, New York J. Math. 6 (2000), 1-20.

[13] A. Kumjian, D. Pask, I. Raeburn, and J. Renault, Graphs, groupoids, and Cuntz-Krieger algebras, J. Funct. Anal. 144 (1997), 505-541.

[14] P. Muhly, J. Renault and D.P. Williams, Equivalence and Isomorphism for groupoid $C^{*}$-algebras, J. Operator Theory 17 (1987), 3-22.

[15] A.L.T. Paterson, Graph inverse semigroups, groupoids and their $C^{*}$-algebras, J. Operator Theory 48 (2002), 645-662.

[16] A.L.T. Paterson, Groupoids, inverse semigroups, and their operator algebras, Birkhäuser Boston Inc., Boston, MA, 1999, xvi+274.

[17] J. Renault, A groupoid approach to $C^{*}$-algebras, Springer, Berlin, 1980, ii +160 .

[18] J. Renault, A. Sims, D.P. Williams and T. Yeend, Uniqueness Theorems for topological higher-rank graph $C^{*}$-algebras, preprint 2012 (arXiv:math/0906.0829).

[19] A.P.W. Sørensen, Geometric classification of simple graph algebras, Ergodic Theory Dynam. Systems. 33 (2013), 1199-1220.

[20] A. Sims and D.P. Williams, Renault's equivalence theorem for reduced groupoid C*-algebras, J. Operator Theory 68 (2012), 101-117.

[21] B. Steinberg, Strong Morita equivalence of inverse semigroups, Houston J. Math. 37 (2011), 895-927.

[22] B. Steinberg, A groupoid approach to discrete inverse semigroup algebras, Adv. Math. 223 (2010), 689-727.

[23] M. Tomforde, Leavitt path algebras with coefficients in a commutative ring, J. Pure Appl. Algebra 215 (2011), 471-484.

[24] S.B.G. Webster, The path space of a directed graph, Proc. Amer. Math. Soc. 142 (2014), $213-225$.

[25] D.P. Williams, Crossed products of $C^{*}$-algebras, Mathematical Surveys and Monographs, 134. American Mathematical Society, Providence, RI, 2007.

[26] T. Yeend, Groupoid models for the $C^{*}$-algebras of topological higher-rank graphs, J. Operator Theory 57 (2007), 95-120.

Lisa Orloff Clark, Department of Mathematics and Statistics, University of Otago, PO Box 56, Dunedin 9054, New Zealand

E-mail address: lclark@maths.otago.ac.nz

Aidan Sims, School of Mathematics and Applied Statistics, University of Wollongong, NSW 2522, Australia

E-mail address: asims@uow.edu.au 